\title{
Do host species evolve a specific response to slave-making ants?
}

\author{
Olivier Delattre ${ }^{1 *}$, Rumsaïs Blatrix ${ }^{2}$, Nicolas Châline ${ }^{1,3}$, Stéphane Chameron ${ }^{1}$, Anne Fédou', Chloé Leroy ${ }^{1}$ \\ and Pierre Jaisson ${ }^{1}$
}

\begin{abstract}
Background: Social parasitism is an important selective pressure for social insect species. It is particularly the case for the hosts of dulotic (so called slave-making) ants, which pillage the brood of host colonies to increase the worker force of their own colony. Such raids can have an important impact on the fitness of the host nest. An arms race which can lead to geographic variation in host defenses is thus expected between hosts and parasites. In this study we tested whether the presence of a social parasite (the dulotic ant Myrmoxenus ravouxi) within an ant community correlated with a specific behavioral defense strategy of local host or non-host populations of Temnothorax ants. Social recognition often leads to more or less pronounced agonistic interactions between non-nestmates ants. Here, we monitored agonistic behaviors to assess whether ants discriminate social parasites from other ants. It is now well-known that ants essentially rely on cuticular hydrocarbons to discriminate nestmates from aliens. If host species have evolved a specific recognition mechanism for their parasite, we hypothesize that the differences in behavioral responses would not be fully explained simply by quantitative dissimilarity in cuticular hydrocarbon profiles, but should also involve a qualitative response due to the detection of particular compounds. We scaled the behavioral results according to the quantitative chemical distance between host and parasite colonies to test this hypothesis.
\end{abstract}

Results: Cuticular hydrocarbon profiles were distinct between species, but host species did not show a clearly higher aggression rate towards the parasite than toward non-parasite intruders, unless the degree of response was scaled by the chemical distance between intruders and recipient colonies. By doing so, we show that workers of the host and of a non-host species in the parasitized site displayed more agonistic behaviors (bites and ejections) towards parasite than toward non-parasite intruders.

Conclusions: We used two different analyses of our behavioral data (standardized with the chemical distance between colonies or not) to test our hypothesis. Standardized data show behavioral differences which could indicate qualitative and specific parasite recognition. We finally stress the importance of considering the whole set of potentially interacting species to understand the coevolution between social parasites and their hosts.

Keywords: Coevolution, Formicidae, Social recognition, Social parasitism, Temnothorax

\section{Introduction}

Parasitism is an interspecific interaction where the host species suffers from the exploitation of its resources by the parasite. Any defense strategy minimizing the impact of the parasite on host fitness is therefore likely to be selected for. In this case, escalation of reciprocal counter-adaptations between the parasite and its host is

\footnotetext{
* Correspondence: olivier.delattre@leec.univ-paris13.fr

'Laboratoire d'Ethologie Expérimentale et Comparée, Université Paris 13, Sorbonne Paris Cité, 99 avenue J.-BClément 93430, Villetaneuse, France Full list of author information is available at the end of the article
}

likely to lead to an "arms race" [1]. This coevolutionary process is considered an important factor that shapes species life history and plays a key role in the geographic mosaic of coevolution through local adaptations [2,3]. Thus, elucidating patterns of local host-parasite interactions helps understanding prominent factors in the evolution of life history traits.

Social parasitism is a complex parasite-host interaction since it not only involves individuals but societies. Mainly found in social bees, wasps and ants, social parasites use the worker force of another social species to

\section{Biomed Central}


rear their own brood [4]. In the case of dulotic species (so called slave-makers), the parasite workers are specialized for conducting raids in a two-step process [5]. First, scouts individually search for potential host nests. When successful, the scout returns to its nest and recruits nestmates to initiate the raid, during which slave-maker ants seize brood and bring it back home. Later, host workers emerging in the parasite nest will be imprinted on and integrated into the mixed colony where they rear the parasite brood, feed and groom the parasite workers, defend the nest against aliens, and even participate in raids. Raids can jeopardize host colony survival [6-8], therefore exerting a strong selection pressure upon the hosts. Reciprocally, there is some evidence that hosts also exert a selection pressure on their parasites in return [9], since resistance by host colonies might prevent enslavement [10]. Coevolutionary processes between dulotic ant species and their hosts then can escalate to an evolutionary arms race $[11,12]$.

In ants, social recognition is based on cuticular hydrocarbon profiles which display qualitative differences between distinct species and quantitative ones between homospecific colonies [13-15]. Since each species is characterized by a qualitatively unique blend of cuticular hydrocarbons [14], an innate mechanism to detect some chemical cues from the parasite could have evolved in host species. In addition, we know that ants can learn to recognize aliens and modify their subsequent behavior in a variety of social contexts [16-18] and social parasites have been proven to modify host behavior in some species $[12,19,20]$.

Myrmoxenus ravouxi is a dulotic social parasite with a wide distribution in Europe ranging from France to Greece [21]. It is known to parasitize several species of the diversified genus Temnothorax [21-23]. Some Temnothorax species are potential hosts for several social parasites [5], while others are never parasitized. Interestingly, the distribution range of the hosts often exceeds the parasite's, leaving some host populations parasite-free. This ant genus thus allows for comparative analysis of defense strategy both between species (host and non-host) and within species (populations with and without parasites). In this study we tested whether the presence of a social parasite within an ant community correlated with a specific behavioral defense strategy of local host or non-host populations. Behavioral responses of colonies to the introduction of parasite (M. ravouxi), homospecific and heterospecific workers were compared between hosts (Temnothorax unifasciatus and T. rabaudi) and non-host (T. nylanderi) species from two sites, one with and one without the parasite. We expected the behavioral response of host species' colonies in the parasitized population to be more aggressive toward parasite intruders than toward other non-parasite intruders.

It is usually assumed that agonistic interactions between ants depend on the dissimilarity between cuticular hydrocarbon profiles [14,24,25], with aggressiveness escalating when dissimilarity increases [26,27]. A new theoretical model recently emerged based on empirical data [28], which suggests that ants may discriminate aliens using the presence of undesirable chemical compounds absent from their nestmates' chemical signature [25], therefore focusing on qualitative differences rather than on global dissimilarity. To separate out quantitative and qualitative components of the chemical recognition, we assessed the chemical quantitative dissimilarity between colonies by calculating the Euclidean distance using chromatogram peaks areas [29-31] for components that were found in all groups, leaving out components that occurred only in some groups and were therefore responsible for qualitative differences between cuticular profiles (see supplementary data). Such methodology amounts to calculating the distance between colonies in a $\mathrm{n}$-dimensional space where all peaks have the same weight $(1 / \mathrm{n})$. This simple calculation is not intended to mirror the actual sensory and cognitive treatment used by ants in social decision-making processes, but it provides us with a way to quantitatively assess chemical distance without referring to any special role for particular compounds. If host species have evolved a specific recognition mechanism toward their parasite, the behavioral response to $M$. ravouxi should not be fully explained simply by overall cuticular profile dissimilarity, but also involve qualitative detection of some specific compounds. The agonistic response of Temnothorax host species, or populations, to the social parasite should therefore fulfill two criterions to be considered specific: a) it should be different from the response to other non-parasite species, and b) it should be at least partially independent of the global dissimilarity between chemical profiles.

\section{Results}

\section{Cuticular hydrocarbon profiles}

The principal coordinate analysis (PCO, Figure 1) clearly sorts out our different populations (cf. Additional file 1). Cuticular hydrocarbon profiles of the colonies of the different populations were significantly different overall (PERMANOVA, pseudo-F $=42.931 P<0.001$ ), and in all pairwise comparisons (PERMANOVA, Pair-wise tests, all $P<0.006$ ). A canonical analysis (data not shown) shows that more than $99.2 \%(117 / 118)$ of our samples were correctly assigned to their respective populations, the only exception being a $T$. unifasciatus colony from Fontainebleau assigned to the Anduze population of the same species. As expected, M. ravouxi 


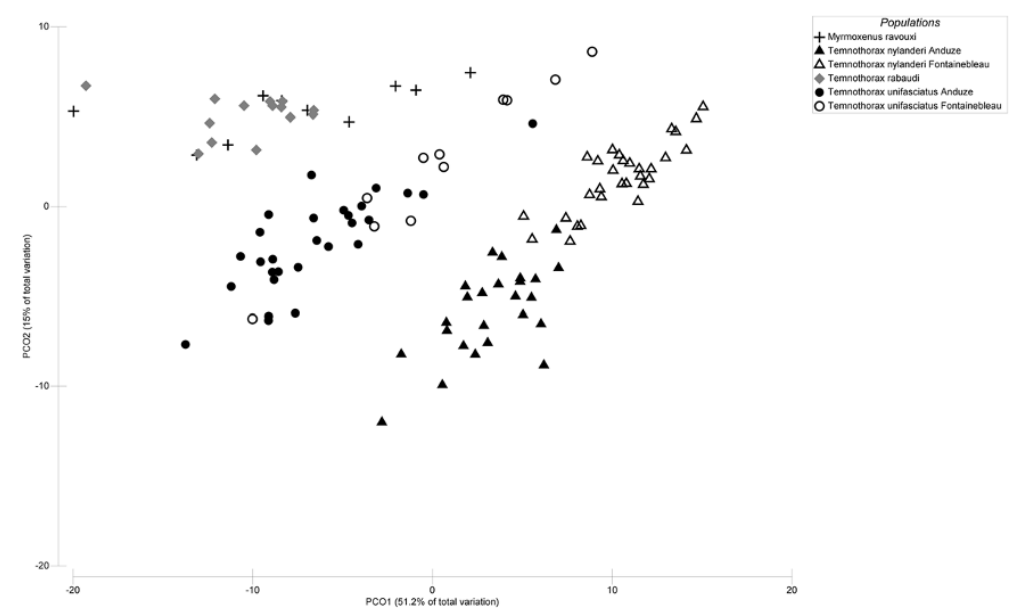

Figure 1 Principal coordinate analysis. Principal coordinate analysis (PCO) based on the resemblance matrix calculated from Euclidean distances between every pair of samples for the 41 peaks present in all populations of M. ravouxi, T. unifasciatus, T. nylanderi and T. rabaudi from Anduze (myr_an, uni_an, nyl_an and rab_an respectively), and of T. unifasciatus and T. nylanderi from Fontainebleau (uni_f and nyl_f respectively).

profiles were close to those of $T$. rabaudi slaves (see mean chemical distances in Additional file 1), but still different, which is consistent with both theoretical and empirical data on mixed species colonies [27,32-34].

\section{Agonistic response of the recipient colonies Bites}

For each population, colonies were more aggressive toward parasites than toward homospecifics (Figure 2; paired permutation tests, $P^{\prime}<0.001$ ).

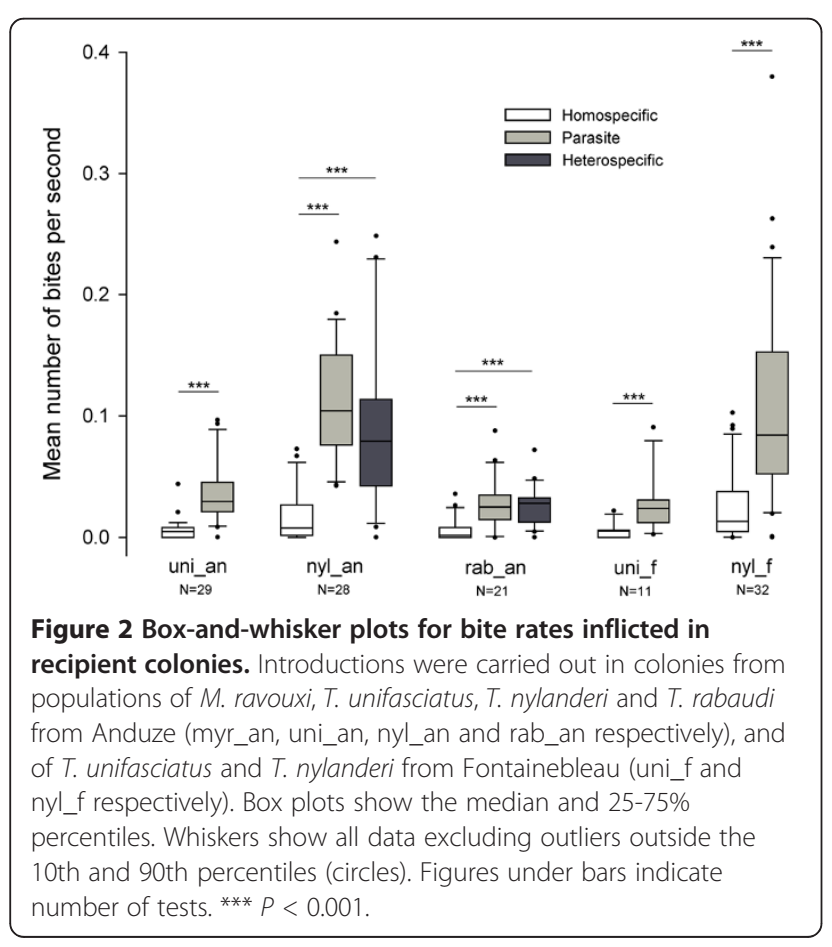

The Kruskal-Wallis analysis showed a significant effect of the "population" factor on the response to introduced homospecific workers (Figure 2; $\mathrm{H}=17.69, P=0.0014$ ). The non-host species ( $T$. nylanderi) was more aggressive in Fontainebleau than in Anduze (permutation test, $P^{\prime}=$ 0.034 ), while other pairwise comparisons were not significant (permutation tests, all $P^{\prime}>0.089$ ).

The "population" factor proved also significant for the colony responses to introduced parasite workers (Figure 2; Kruskal-Wallis, $\mathrm{H}=55.77, P<0.001)$. The non-host species was significantly more aggressive than the other species at both sites (permutation tests, in Anduze, compared with T. unifasciatus: $P^{\prime}<0.001$; T. rabaudi: $P^{\prime}<$ 0.001; in Fontainebleau, T. unifasciatus: $P^{\prime}=0.007$ ). $T$. nylanderi colonies from Anduze were more aggressive toward parasite workers than T. nylanderi colonies from Fontainebleau (permutation test, $P^{\prime}=0.0056$ ).

Introduction of a non-parasite heterospecific worker was performed in colonies of T. nylanderi and T. rabaudi from the parasitized site (Anduze) in order to test whether ants were able to discriminate the parasite from another heterospecific worker. For both species, the responses toward parasites, homospecifics and heterospecifics were significantly different (Figure 3; Kruskal-Wallis, T. nylanderi: $\mathrm{H}=44, P<0.001 ; T$. rabaudi: $\mathrm{H}=19.02$, $P<0.001)$. For both species the agonistic response was lowest toward homospecific intruders (Figure 3; permutation tests, $P^{\prime}<0.001$ ) but was not significantly different toward parasites or heterospecific non-parasites (permutation tests, $P^{\prime}=0.108$ and $P^{\prime}=0.577$, respectively).

\section{Bites (scaled by chemical distance)}

For each population except $T$. rabaudi from Anduze and T. nylanderi from Fontainebleau (Figure 3; paired 


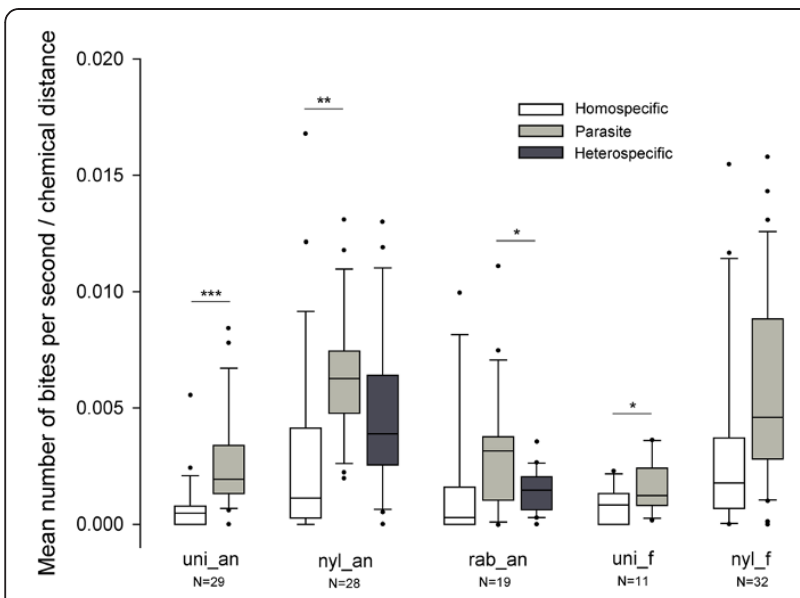

Figure 3 Box-and-whisker plots for bite rates inflicted in recipient colonies scaled by chemical distance. We scaled the mean bite rates by chemical distance between the intruders and the recipient colonies from populations of $M$. ravouxi, T. unifasciatus, $T$. nylanderi and T. rabaudi from Anduze (myr_an, uni_an, nyl_an and rab_an respectively), and of $T$. unifasciatus and $T$. nylanderi from Fontainebleau (uni_f and nyl_f respectively). Box plots show the median and $25-75 \%$ percentiles. Whiskers show all data excluding outliers outliers outside the 10th and 90th percentiles (circles). Figures under bars indicate number of tests. ${ }^{*} P<0.05,{ }^{* *} P<0.01$, *** $P<0.001$

permutation tests, $P^{\prime}=0.342$ and $P=0.15$ ), colonies were significantly more aggressive toward parasites than toward homospecifics (Figure 3; paired permutation tests, all $P<0.011$ ).

Colony responses to introduced homospecific workers significantly differed between populations (Figure 3; Kruskal-Wallis, $\mathrm{H}=17.88, P=0.0013)$. The non-host species ( $T$. nylanderi) was more aggressive than $T$. unifasciatus in Anduze (Permutation test, $P^{\prime}=0.028$ ) but not in Fontainebleau $\left(P^{\prime}=0.317\right)$. We found no differences between sites for either T. unifasciatus or $T$. nylanderi $\left(P^{\prime}=1\right.$ and $P^{\prime}=1$, respectively).

Colony responses to introduced parasite workers also differed significantly between populations (Figure 3; Kruskal-Wallis, $\mathrm{H}=35.5, P<0.001)$. The non-host species was significantly more aggressive than the other species in both sites (permutation tests, in Anduze, compared with $T$. unifasciatus: $P^{\prime}<0.001 ;$ T. rabaudi: $P^{\prime}=0.0016$; in Fontainebleau, $T$. unifasciatus: $P^{\prime}=$ 0.022). The response of non-host against parasites was not significantly different between sites (permutation test, $\left.P^{\prime}=1.1268\right)$.

Introduction of a non-parasite heterospecific worker was performed in colonies of $T$. nylanderi and $T$. rabaudi from the parasitized site (Anduze). For both species, the responses toward parasites, homospecifics and heterospecifics were significantly different (Figure 3; Kruskal-Wallis, T. nylanderi: $\mathrm{H}=18.71, P<0.001 ; T$. rabaudi: $\mathrm{H}=9.082, P=0.011)$. $T$. nylanderi colonies were marginally more aggressive toward parasites than toward heterospecific non-parasites (Figure 3; permutation test, $\left.P^{\prime}=0.086\right)$. No statistical difference could be observed for the agonistic response of $T$. rabaudi between homospecific heterocolonial intruders and heterospecific ones, be they parasites or not (all $P^{\prime}>0.342$ ). However, $T$. rabaudi were significantly more aggressive toward parasites than toward heterospecific non-parasite intruders (permutation test, $P^{\prime}=0.018$ ).

\section{Ejections}

Ejection rates of homospecific intruders were not significantly different between populations (Figure 4, FisherFreeman-Halton test, $\left.P^{\prime}=0.095\right)$. On the contrary, ejection rates of parasites were significantly different between populations (Figure 4, Fisher-Freeman-Halton test, $\left.P^{\prime}=0.002\right)$ : the non-host species (T. nylanderi) from the parasitized site (Anduze) tended to display this behavior more often than other populations (Fisher tests, comparison with $T$. unifasciatus from Anduze: $P^{\prime}=$ 0.052; T. rabaudi from Anduze: $P^{\prime}=0.094 ; T$. nylanderi from Fontainebleau: $P^{\prime}=0.057$ ) but it was not significant when compared to $T$. unifasciatus from Fontainebleau $\left(P^{\prime}=0.381\right)$. More importantly, $T$. nylanderi from Anduze was the only population in which parasites were ejected significantly more often than homospecific intruders (ejection rates respectively 0.72 and 0.26 , Fisher test, $\left.P^{\prime}=0.011\right)$; it also ejected parasite workers significantly more frequently than heterospecifics (ejection rates respectively 0.72 and 0.24 , Fisher test, $P^{\prime}=0.012$ ),

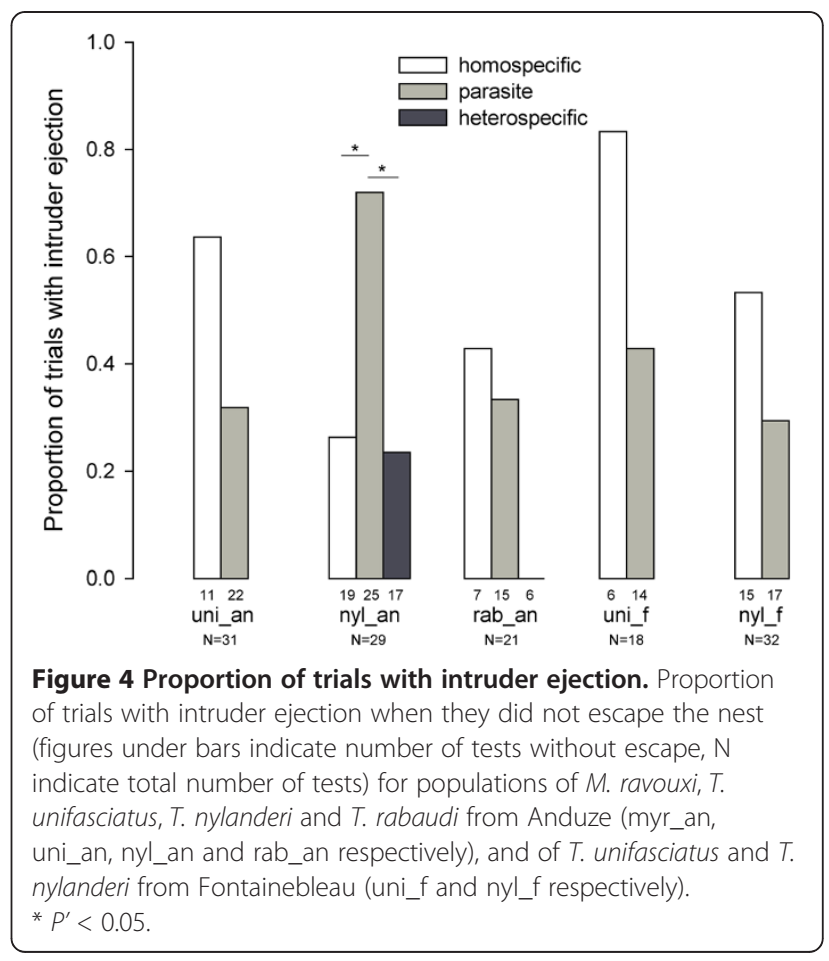


whose ejection rate did not differ from homospecifics' (Fisher test, $P^{\prime}=1$ ).

\section{Discussion}

In Anduze, all four species could be discriminated using their cuticular hydrocarbon profiles. Profiles of M. ravouxi were very close to those of the main host, $T$. rabaudi, suggesting integration of host components into the chemical profile of the parasite. This mechanism has already been shown in dulotic ants [35-40]. However, in our study, the profiles of the parasite and its main host were distinct (cf. Additional file 1). GC-MS analyses also showed some qualitative differences between the parasite and its host (cf. Additional file 2). Chemical analyses thus prove the potentiality for hosts to recognize the parasite and therefore to display a specific defense behavior, even if the chemical closeness of the social parasite might also make recognition more difficult.

With the raw data, all studied populations reacted more aggressively to allospecific intruders (be they parasites or not) than to homospecific heterocolonial ones. The non-host species T. nylanderi, both from the parasitized (Anduze) and non-parasitized site (Fontainebleau), was more aggressive toward parasite intruders than the other species.

Neither $T$. rabaudi nor $T$. nylanderi from Anduze reacted more aggressively to the parasite than to a nonparasite heterospecific intruder. Response of T. unifasciatus to the parasite was not significantly different between Anduze and Fontainebleau (the parasite-free site). Thus, we could not detect any clear-cut effect of the presence of the parasite in the ant community on the defense strategy of the two host species.

This pattern could be explained by the fact that, in this host-parasite local interaction, the parasite species is leading the arms race and offsets any specific defense through mimetic odor [35,41] or propaganda allomone [42]. Moreover, as opportunist $M$. ravouxi slave-making ants can use more than five host species [23,43], it may shift from one host species to another in the same site, for example as a consequence of any counter adaptations from its host species. An alternative hypothesis would be that the cost of being parasitized is lower than the cost of a specific defense which could increase nestmate recognition errors [44] and decrease colony fitness.

The raw results show that host and non-host species are not specifically more aggressive toward parasite than non parasite intruders, but the non-host species display more aggressiveness toward these intruders than the host species. T. nylanderi populations are sometimes very dense $[45,46]$ and competition for nest sites may cause colony fusion. These conflicts and subsequent intraspecific parasitism may select for a higher level of aggressiveness in dense populations of this species. In
Fontainebleau, where we found 1-3 colonies per square meter, $T$. nylanderi display a higher degree of aggressiveness toward homospecific intruders. We thus may hypothesize that in this site, T. nylanderi colonies lowered their tolerance threshold in response to intraspecific competition.

Interestingly, T. nylanderi colonies from the parasitized site of Anduze bit more often parasite intruders than T. nylanderi colonies from the non-parasitized site of Fontainebleau. However, the difference between the medians is very small $(0.104$ vs. 0.0842$)$, and thus we cannot conclusively interpret them as a product of local adaptation processes.

In order to investigate a potential specific response to the slave-making parasite $M$. ravouxi parasite, which could not be fully explained by the global chemical dissimilarity, we also compared agonistic responses when scaled according to the quantitative chemical distance between host and intruder colonies. A slightly different picture arises from this second analysis. Differences between homospecific and parasite introductions remain significant, with the exception of $T$. rabaudi colonies from Anduze and T. nylanderi from Fontainebleau, which are not significantly more aggressive toward parasite than toward homospecific intruders.

T. rabaudi host colonies from the parasitized population (Anduze) were more aggressive toward parasite than non-parasite heterospecific workers. T. nylanderi colonies displayed a stronger agonistic response while biting more often parasite than homospecific intruders and showing a tendency to be more aggressive toward parasite than non-parasite heterospecific intruders after standardization $\left(P^{\prime}=0.086\right)$. Host species colonies did not show more aggressiveness toward non-parasite heterospecifics than toward homospecifics intruders in our second analysis. Parasite intruders were extracted from T. rabaudi parasitized colonies and were thus more likely to bear some of the specific chemical cues this host species displays. It could explain why $T$. rabaudi colonies did not show a higher aggressiveness toward the parasite workers when we scaled down the importance of quantitative differences. Still, free-living host colonies did show a higher agonistic response to parasite than to non-parasite heterospecific intruders. We thus suggest that the more aggressive response to parasite than to heterocolonial homospecifics or non-parasite heterospecifics after standardization indicates that ant aggressiveness does not solely increase linearly with global chemical distance. Pamminger and collaborators [20] showed that aggressive reaction of Temnothorax longispinosus colonies against non-parasite congeneric workers transiently rose after the introduction of a dead slavemaking Protomognatus americanus worker, when the introduction of a dead congeneric worker had no visible 
consequence. They concluded that $T$. longispinosus specifically reacted to the parasite presence in an adaptive way by lowering their general tolerance threshold, since Temnothorax enslaved workers join parasite workers during raids and fiercely attack host colonies. Parasite presence indeed reliably signals a forthcoming raid, which is not the case for congeneric workers that can belong to non-parasite colonies. We thus may hypothesize that Temnothorax ants rely on qualitative analysis of the cuticular profiles to discriminate parasite from non-parasite species in parasitized populations. This could result from ants weighting more some compounds (be they specific to the parasite or not), either because of the cognitive processes used to match the perceived cuticular profile and the internal template $[14,44]$, or because sensory systems are more sensitive to qualitative differences than quantitative ones (some heterospecific compounds are absent of the internal template, while homospecific profiles simply differ in a quantitative way) [26], or both, since these mechanisms are not mutually exclusive.

In some behavioral tests, recipient workers also ejected the intruder. Such a behavior is probably not very efficient in preventing raids in host colonies, because parasite workers could easily return to the raided nest. However, it could have been selected as a ritualized fighting behavior [47-49] in non-host species since it allows conflict resolution without casualties (the intruder usually put up little resistance). The ejection strategy could thus have been selected to prevent fights in cases where a social parasite scout enters a colony from the non-host colony. $M$. ravouxi scouts also have no interest in engaging in fights in non-host species colonies. Slavemaking ants may discriminate host from non-host species colonies, using for example a recognition template based on innate or experience-induced preferences $[50,51]$. They could then also actively avoid conflicts with non-host workers by inducing ejection behaviors. Nonetheless, in order to limit the interaction effect on ejections by recipient colonies, we use proportion of ejections only where the intruders did not escape. Moreover, T. nylanderi from the parasitized site of Anduze showed a higher rate of ejection of parasite intruders than of non-parasite heterospecifics or heterocolonial homospecifics, while ejection rates displayed by $T$. $n y l a n d e r i$ in the parasite-free site (Fontainebleau) were not different between parasite and homospecific intruders. Besides, T. nylanderi ejected the parasite more often in Anduze than in Fontainebleau. Taken together, these results show that $T$. nylanderi from the parasitized site displayed a specific response to the social parasite, even though it is not used as a host. The specific response consisted in a higher rate of a ritualized behavior and marginally more agonistic interactions (respectively ejections and bites). T. nylanderi has never been observed as host, but our results suggest potential interactions between the two species. This is obviously difficult to verify because raids are rarely observed in the field. Thus, we cannot know if the specific behavior has been locally selected per se, or if it results from the learning of parasite odor through experience prior to colony collection. To tell apart the respective roles of local selection and learning capacities, similar experiments should be conducted with $T$. nylanderi colonies reared in the lab from founding queens from Anduze.

\section{Conclusions}

Our results did not show unequivocal evidence of a host behavioral defense that would be specific to the social parasite $M$. ravouxi. Nonetheless, standardization of agonistic behaviors by chemical distance between intruder and receiver colonial signatures shows a higher aggressiveness toward the parasite than toward a non-parasite heterospecific intruder for the host species, which could result from a coevolutionary arms race [7,8,10,11,37]. We do not know how ants' decision rules are implemented, i.e. following qualitative or quantitative dissimilarity of chemical cues. Ants certainly may adjust their behavior according to the absence/presence of some particular chemical cues when facing conspecifics [51,52]. So, we think that standardization of behavioral results by chemical distance between colonies in social insects may bring new insights on the discrimination mechanisms towards sympatric species of social parasites. In this aspect, our methodology shows a pattern of host resistance. Moreover, we show for the first time that a species never recorded as host ( $T$. nylanderi) can also display a specific behavioral defense to a slave-making ant. The specific defense included higher rates of bites and ritualized ejections of parasite workers. It remains to be tested whether $M$. ravouxi attempts raids on $T$. nylanderi in the field. Our results lead us to stress the importance of considering the whole set of potentially interacting species to fully understand the geographic mosaic of host-parasite interactions $[53,54]$.

\section{Materials and methods}

\section{Study species}

The parasite species $M$. ravouxi was collected in Anduze (N44'3' E3 $\left.{ }^{\circ} 59^{\prime}\right)$. T. unifasciatus and T. rabaudi are common hosts of $M$. ravouxi. T. unifasciatus colonies were collected both in the Anduze site and in Fontainebleau (N48 24' E2 $42^{\prime}$ ), which is outside the distribution area of $M$. ravouxi. T. rabaudi could only be found in Anduze, where it is the most frequent host for $M$. ravouxi (some parasite colonies where found with $T$. unifasciatus slave workers). Last, we collected T. nylanderi colonies in both the Anduze and Fontainebleau sites. To the best of our knowledge, T. nylanderi has 
never been recorded as a host for any social parasite (the records of Bernard [55,56] could not be confirmed by Buschinger et al. [57]); however, it could be found in Anduze in sympatry with the slave-making $M$. ravouxi. In Fontainebleau, T. nylanderi was very frequent, with densities of almost 2 or 3 colonies per square meter. This could limit the number of available nest sites and cause intraspecific colony fusions and parasitism in this species [58].

Throughout the rest of this manuscript refer to "populations" for colonies of a given species originating from the same site: T. unifasciatus from Anduze (uni_an, $\mathrm{N}=29$ ), T. unifasciatus from Fontainebleau (uni_f, $\mathrm{N}=11$ ), T. nylanderi from Anduze (nyl_an, $\mathrm{N}=28$ ), $T$. nylanderi from Fontainebleau (nyl_f, $\mathrm{N}=32$ ), T. rabaudi from Anduze (rab_an, $\mathrm{N}=21$ ), and $M$. ravouxi from Anduze (myr_an, $\mathrm{N}=13$ ). The latter were only used as a source of parasite workers to introduce in the free-living tested colonies. These parasite workers were thus all extracted from $T$. rabaudi parasitized colonies. We also refer to T. nylanderi as "non-host species", T. unifasciatus and T. rabaudi as "host species", Fontainebleau as "nonparasitized site" and Anduze as "parasitized site".

Colonies were all queen-right and reared in the lab in artificial nests made of two microscope slides superimposed and kept $1 \mathrm{~mm}$ apart from each other using a thin piece of linoleum. They were fed with honey and fruit flies. The experiments took place in the spring, from April to May, after a three-month wintering period at $8^{\circ} \mathrm{C}$. The colonies were collected at the end of the previous summer. The temperature during the experiments was around $24^{\circ} \mathrm{C}$.

\section{Characterizing dissimilarity between cuticular hydrocarbons profiles}

The cuticular hydrocarbons profile was characterized for each colony by soaking 10 workers in $50 \mu$ l of pentane for ten minutes. An aliquot of $2 \mu \mathrm{l}$ was analyzed using gas-chromatography with flame ionization detection (GC-FID) on a Varian 3900 instrument equipped with a split/splitless injector and a DB5 fused silica capillary column ( $30 \mathrm{~m} \times 0.32 \mathrm{~mm}, 0.25 \mu \mathrm{m}$ film thickness), with helium as carrier gas at a flow rate of $28.57 \mathrm{cc} / \mathrm{sec}$. The temperature was maintained at $100^{\circ} \mathrm{C}$ for $5 \mathrm{~min}$, raised at $3^{\circ} \mathrm{C} / \mathrm{min}$ to $300^{\circ} \mathrm{C}$ and held constant for $10 \mathrm{~min}$. Peak areas were calculated using the Varian system control. Peaks were later identified using a GC-MS, allowing us to exclude contaminants from analyses. Analyses were carried out on a Agilent $5975 \mathrm{C}$ inert XL with chromatograph GC system 7890A of Agilent with a split-splitless injector and a fused-silica capillary column (30 meters long with a diameter of $250 \mu \mathrm{m}$ ) with a $0.25 \mu \mathrm{m}$ polydimethylsiloxane coating. The carrier gas was helium (99.99\%) and the column temperature program was $100^{\circ} \mathrm{C}$
(5 $\mathrm{min})$ and $3^{\circ} / \mathrm{min}$ to $300^{\circ} \mathrm{C}(10 \mathrm{~min})$. The injection port temperature was $280^{\circ} \mathrm{C}$. Total ion chromatograms and mass spectra were recorded in the electron impact ionization mode at $70 \mathrm{eV}$. The transfer line and the source temperature were maintained at $230^{\circ} \mathrm{C}$. Compound identifications were based on retention times and comparison with published data [59]. In a first step, peaks displaying an area above or equal to $1 \%$ of the total area in at least 10 samples (66 peaks) were selected. In a second step, we discarded all peaks that were not present in all populations, to focus on quantitative rather than qualitative differences between chemical profiles; this lead to a final selection of 41 peaks. Euclidean distances between every pair of samples were calculated to produce a resemblance matrix. A principal coordinate analysis (PCO) using this matrix was then performed to sort out the cuticular hydrocarbon profiles of the colonies. We compared the colonial profiles of our populations with a single factor PERMANOVA $[60,61]$ using 9999 permutations, first to detect overall differences and then in one-by-one tests between the populations. Statistical analyses on the chemical profiles were carried out with the PERMANOVA+ V1.0.2 addon package [62] of PRIMER V6.1.12 [63]. The mean distance between populations' centroids was also calculated to illustrate the calculated quantitative similarity between them (Additional file 1).

\section{Behavioral observations}

The tests consisted in the introduction of an alien worker inside a colony, slightly removing the upper side of the nest to obtain a small opening on one side of the colony and to introduce the intruder directly inside it. All colonies were tested twice, using a parasite (M. ravouxi) and a conspecific heterocolonial intruder (from the same site). M. ravouxi alien workers came from parasitized colonies of $T$. rabaudi $(\mathrm{N}=13)$. For $T$. rabaudi and T. nylanderi from Anduze, a third test was performed using a heterospecific, non-parasite intruder ( $T$. rabaudi for $T$. nylanderi and vice versa, both from the same site and from free-living colonies). The sequence of introductions (parasite, heterocolonial and heterospecific non-parasite) was randomized between colonies to avoid any order effect. We used only local conspecifics or heterospecifics for introductions, except for $M$. ravouxi intruders, as this species could only be found in the south of France. We did so because coevolutionary processes, which could lead to pattern of specific resistance, are obviously likely to appear locally [2].

The agonistic response of the recipient colony was assessed by recording bites and ejection of the intruder by resident ants (see definitions below) until the alien worker left the nest, and in the few cases this did not happen within 30 minutes the test was terminated. Test duration was thus variable, but lower than 30 minutes. 


\section{Bites}

We compared the agonistic response of colonies during the tests using the bite rates (number of bites divided by test duration).

We also performed a second analysis of our agonistic response by dividing these rates by the chemical distance between the intruder and the recipient colony, to test for a specific recognition on qualitative chemical cues. The rationale here is that a specific recognition mechanism would lead to higher levels of agonistic interactions towards the parasite than towards other intruders, even after getting rid of their global chemical distance with the recipient colony.

The chemical distance D was defined as the Euclidean distance between chemical profiles [30-32]:

$$
D(x, y)=\sqrt{\sum_{i}(x i-y i)^{2}}
$$

where $\mathrm{x}$ and $\mathrm{y}$ refer to the two colonies, $\mathrm{x}_{i}$ and $\mathrm{y}_{i}$ to the relative areas of peak $i$ for colonies $\mathrm{x}$ and $\mathrm{y}$ respectively. When the chemical profile of a colony was not available (for technical reasons), we used the mean chemical profile of the corresponding population. This was the case for 5 homospecific tests with $T$. nylanderi from Fontainebleau, 11 homospecific tests with T. unifasciatus from Fontainebleau, 14 homospecific and 7 non-parasite heterospecific tests with $T$. rabaudi from Anduze. In our second analysis, we had to discard two $T$. rabaudi colonies confronted in a test (recipient/intruder) because we did not know their chemical profile.

Comparisons were performed using Kruskal-Wallis and permutation tests with general scores for paired samples [64]. We compared populations from different species within the same site and populations from the same species between sites. Other comparisons were considered irrelevant, i.e. different species from different sites. In order to use paired permutation tests between the two (or three) behavioral tests for each population (using the sequential Bonferroni-Holm correction procedure when necessary [65]), the few recipient colonies that had performed only one type of test were discarded from the analysis (respectively 7 and 2 for T. unifasciatus from Fontainebleau and Anduze, and 1 for T. nylanderi from Anduze).

\section{Ejections}

Apart from bites we also observed, in some trials, recipient ants grasping the intruder by an appendage and dragging it outside of the nest, a behavior we referred to as "ejection". Test outcomes could thus be of three types: the intruder escaped, it was ejected by residents or it could stay in the nest for the whole observation period. To assess the resident propensity to eject intruders, we calculated the proportion of ejections over the tests where the intruder did not escape.

All comparisons were done using Fisher or FisherFreeman-Halton tests [66]. We used the sequential Bonferroni-Holm correction procedure [65] when necessary, and adjusted p-values are noted $P^{\prime}$ (they should be compared to the standard 0.05 significance threshold) [67].

All statistical tests were performed with StatXact (Cytel Studio, version 8.0.0, 2007).

\section{Additional files}

Additional file 1: Distances between centroids. Distance between centroids for the chemical profiles of populations of M. ravouxi, T. nylanderi from Anduze and Fontainebleau, T. rabaudi and T. unifasciatus from Anduze and Fontainebleau.

Additional file 2: Identified cuticular hydrocarbons of species' chemical profiles. Mean percentages $( \pm S . D)$ of the different compounds in the chemical profiles of M. ravouxi (M. rav), T. unifasciatus from Anduze (T. uni_And) and Fontainebleau (T. uni_Font), T. rabaudi (T. rab) and T. nylanderi from Anduze (T. nyl_And) and Fontainebleau (T. nyl_Font). Compounds were identified using GC-MS. Unidentified hydrocarbons are marked with *.

\section{Abbreviations}

Myr: an stands for Myrmoxenus ravouxi from Anduze; nyl: an for Temnothorax nylanderi from Anduze site; nyl: f for Temnothorax nylanderi from Fontainebleau; rab: an for Temnothorax rabaudi from Anduze; uni: an for Temnothorax unifasciatus from Anduze and uni_f for Temnothorax unifasciatus from Fontainebleau.

\section{Competing interests}

The author(s) declare that they have no competing interests.

\section{Authors' contributions}

OD participated in statistical, chromatography and behavioral analysis and wrote the manuscript. RB conceived the study, performed the GC analysis, participated in statistical, chromatography and behavioral analysis and helped to draft the manuscript. NC participated in the GC-MS and behavioral analysis and helped to draft the manuscript. SC performed the GC analysis, participated in statistical, chromatography and behavioral analysis and helped to draft the manuscript. AF carried out the behavioral assays. CL carried out the GC-MS analysis and helped to identify chemical compounds. PJ participated in the design and coordination of the study. All authors read and approved the final manuscript.

\section{Acknowledgements}

The authors wish to thank Patrizia d'Ettorre and two anonymous referees for helpful comments to improve this manuscript, Xavier Espadaler for identification of species and Francesco Bonadonna and Ronara De Souza Ferreira for suggestions on the statistical analysis. Olivier Delattre was supported by the French Ministry of Research. Nicolas Châline and Stéphane Chameron were supported by the ANR project SEUILS ANR-09-JCJC-0031.

\section{Author details}

'Laboratoire d'Ethologie Expérimentale et Comparée, Université Paris 13, Sorbonne Paris Cité, 99 avenue J.-BClément 93430, Villetaneuse, France. ${ }^{2}$ Centre d'Ecologie Fonctionnelle et Evolutive, Centre National de la Recherche Scientifique, 1919 route de Mende, F-34293, Montpellier cedex 5 France. ${ }^{3}$ Departamento de Biologia, FFCLRP, Universidade de São Paulo (USP), Ribeirão Preto, SP, Brazil.

Received: 23 December 2011 Accepted: 17 December 2012

Published: 31 December 2012 


\section{References}

1. Van Valen L: A new evolutionary law. Evol Theor 1973, 10:71-74.

2. Thompson JN: The coevolutionary process. Chicago: The University of Chicago Press; 1994

3. Thompson JN: The geographic mosaic of coevolution. Chicago: The University of Chicago Press; 2005.

4. Hölldobler B, Wilson EO: The ants. Harvard University Press: Cambridge, Mass; 1990.

5. Buschinger A, Ehrhardt W, Winter U: The organization of slave raids in dulotic ants a comparative study (Hymenoptera; Formicidae). Zeit Tierpsychol 1980, 53:245-264.

6. Foitzik S, Herbers JM: Colony structure of a slavemaking ant. II. Frequency of slave raids and impact on the host population. Evolution 2001, 55:316-323.

7. Hare JF, Alloway TM: Prudent Protomognathus and despotic Leptothorax duloticus: differential costs of ant slavery. Proc Nat Acad Sci USA 2001, 98:12093-12096

8. Johnson CA, Herbers JM: Impact of parasite sympatry on the geographic mosaic of coevolution. Ecology 2006, 87:382-394.

9. Achenbach A, Foitzik S: First evidence for slave rebellion: enslaved ant workers systematically kill the brood of their social parasite Protomognathus americanus. Evolution 2009, 63:1068-1075.

10. Foitzik S, Deheer CJ, Hunjan DN, Herbers JM: Coevolution in host-parasite systems: behavioural strategies of slave-making ants and their hosts. Proc Roy Soc B Biol Sci 2001, 268:1139-1146.

11. Foitzik S, Fischer B, Heinze J: Arms-races between social parasites and their hosts: Geographic patterns of manipulation and resistance. Behav Ecol 2003, 14:80-88.

12. Brandt $\mathrm{M}$, Foitzik S, Fischer-Blass B, Heinze J: The coevolutionary dynamics of obligate ant social parasite systems-between prudence and antagonism. Biol Rev Camb Philos Soc 2005, 80:251-267.

13. Lahav S, Soroker V, Hefetz A, Vander Meer RK: Direct behavioral evidence for hydrocarbons as ant recognition discriminators. Naturwissenschaften 1999, 86:246-249.

14. Lenoir A, Fresneau D, Errard C, Hefetz A: Individuality and colonial identity in ants: the emergence of the social representation concept. In Information processing in social insects. Edited by Detrain C, Deneubourg JL, Pasteels J. Basel: Birkhauser Verlag; 1999:219-237.

15. d'Ettorre P, Lenoir A: Nestmate recognition. In Ant Ecology. Edited by Lach L, Parr C, Abbott K. Oxford, UK: Oxford University Press; 2009:194-209. references 319-383.

16. Martin SJ, Vitikainen E, Drijfhout FP, Jackson D: Conspecific ant aggression is correlated with chemical distance, but not with genetic or spatial distance. Behav Genet 2012, 42:323-331.

17. Knaden $M$, Wehner R: Nest defense and conspecific enemy recognition in the desert ant Cataglyphis fortis. J Insect Behav 2003, 16:717-730.

18. Sanada-Morimura S, Minai M, Yokoyama M, Hirota T, Satoh T, Obara Y: Encounter-induced hostility to neighbors in the ant Pristomyrmex pungens. Behav Ecol 2003, 14:713-718.

19. d'Ettorre P, Brunner E, Wenseleers T, Heinze J: Knowing your enemies: seasonal dynamics of host-social parasite recognition.

Naturwissenschaften 2004, 91:594-597.

20. Pamminger T, Scharf I, Pennings PS, Foitzik S: Increased host aggression as an induced defense against slave-making ants. Behav Ecol 2011, 22:255-260.

21. Buschinger A, Winter U: Population studies of the dulotic ant, Epimyrma ravouxi, and the degenerate slavemaker, E. kraussei (Hymenoptera: Formicidae). Entomol Gen 1983, 8:251-266.

22. Buschinger A: Evolution, speciation, and inbreeding in the parasitic ant genus Epimyrma (Hymenoptera, Formicidae). J Evol Biol 1989, 2:265-283.

23. Buschinger A: Socially parasitic formicoxenine ants from Western Europe - a review (Hymenoptera, Formicidae). In Proceedings of the International Colloquia on Social Insects. Volume 3-4. Edited by Kipyatkov VE. St. Petersburg: Russian Language Section of the IUSSI, Socium; 1997:1-9.

24. Ichinose $\mathrm{K}$, Cerda $\mathrm{X}$, Jean-Philippe $\mathrm{C}$, Lenoir A: Detecting nestmate recognition patterns in the fission-performing ant Aphaenogaster senilis: A comparison of different indices. J Insect Behav 2005, 18:633-650

25. Guerrieri FJ, Nehring V, Jorgensen CG, Nielsen J, Galizia CG, d'Ettorre P: Ants recognize foes and not friends. Proc Roy Soc B Biol Sci 2009, 276:2461-2468.

26. Vander Meer RK, Morel L: Nestmate recognition in ants. In Pheromone communication in social insects. Edited by Vander Meer RK, Breed M, Winston M, Espelie KE. Boulder, CO: Westview Press; 1998:368. 79-103.
27. Errard C, Hefetz A, Jaisson P: Social discrimination tuning in ants: template formation and chemical similarity. Behav Ecol Sociobiol 2006, 59:353-363.

28. van Zweden JS, d'Ettorre P: Nestmate recognition in social insects and the role of hydrocarbons. In Insect Hydrocarbons: Biology, Biochemistry and Chemical Ecology. Edited by Blomquist GJ, Bagnères A. Cambridge: Cambridge University Press; 2010:222-243.

29. Boulay R, Cerdá X, Simon T, Roldan M, Hefetz A: Intraspecific competition in the ant Camponotus cruentatus: should we expect the 'dear enemy' effect? Anim Behav 2007, 74:985-993.

30. Martin SJ, Jenner EA, Drijfhout FP: Chemical deterrent enables a socially parasitic ant to invade multiple hosts. Proc Roy Soc B Biol Sci 2007, 274:2717-2721.

31. Vogel V, Pedersen JS, d'Ettorre P, Lehmann L, Keller L: Dynamics and genetic structure of Argentine ant supercolonies in their native range. Evolution 2009, 63:1627-1639.

32. Hefetz A, Errard C, Cojocaru M: The occurrence of heterospecific substances in the postpharyngeal gland secretion of ants reared in mixed species colonies (Hymenoptera: Formicidae). Naturwissenschaften 1992, 79:417-420.

33. Errard C: Development of interspecific recognition behavior in the ants Manica rubida and Formica selysi (Hymenoptera: Formicidae) reared in mixed-species groups. J. Insect Behav 1994, 7:83-99.

34. Errard C, Hefetz A: Label familiarity and discriminatory ability of ants reared in mixed groups. Insect Soc 1997, 44:189-198.

35. Yamaoka R: Chemical ecology of social insects. [in Japanese]. Nippon Nogeikagaku Kaishi = J Agr Chem Soc Jap 1990, 64:1749-1753 [Rev. Appl. Entomol. A 1779: 10390].

36. Bonavita-Cougourdan $A$, Rivière $G$, Provost $E$, Bagnères $A G$, Roux $M$, Dusticier G, Clément JL: Selective adapation of the cuticular hydrocarbon profiles of the slave-making ants Polyergus rufescens Latr. and their Formica rufibarbis Fab. and F. Cunicularia Latr. slaves. Comp Biochem Physiol B 1996, 113:313-329.

37. Bonavita-Cougourdan A, Bagnères AG, Provost E, Dusticier G, Clément JL: Plasticity of the cuticular hydrocarbon profile of the slave-making ant Polyergus rufescens depending on the social environment. Comp Biochem Physiol B 1997, 116:287-302.

38. d'Ettorre P, Mondy N, Lenoir A, Errard C: Blending in with the crowd: social parasites integrate into their host colonies using a flexible chemical signature. Proc Roy Soc B Biol Sci 2002, 269:1911-1918.

39. Bauer S, Böhm M, Witte V, Foitzik S: An ant social parasite in-between two chemical disparate host species. Evol Ecol 2010, 24:317-332.

40. Ruano F, Devers S, Sanllorente O, Errard C, Tinaut A, Lenoir A: A geographical mosaic of coevolution in a slave-making host-parasite system. J Evol Biol 2011, 24:1071-1079.

41. Franks N, Blum M, Smith RK, Allies AB: Behavior and chemical disguise of cuckoo ant Leptothorax kutteri in relation to its host Leptothorax acervorum. J Chem Ecol 1990, 16:1431-1444.

42. Allies $A B$, Bourke AFG, Franks NR: Propaganda substances in the cuckoo ant Leptothorax kutteri and the slave-maker Harpagoxenus sublaevis. J Chem Ecol 1986, 12:1285-1293.

43. Seifert B: Die Ameisen Mittel- und Nordeuropas. Tauer: Lutra Verlags- und Vertriebsgesellschaft; 2007.

44. Reeve HK: The evolution of conspecific acceptance thresholds. Am Nat 1989, 133:407-435.

45. Heinze J, Foitzik S, Hippert A, Hölldobler B: Apparent dear-enemy phenomenon and environment-based recognition cues in the ant Leptothorax nylanderi. Ethology 1996, 102:510-522.

46. Foitzik S, Sturm H, Pusch K, d'Ettorre P, Heinze J: Nestmate recognition and intraspecific chemical and genetic variation in Temnothorax ants. Anim Behav 2007, 73:999-1007.

47. Maynard-Smith J, Price GR: The logic of animal conflict. Nature 1973, 246:15-18.

48. Maynard-Smith J: Game theory and the evolution of behaviour. Proc Roy Soc B Biol Sci 1979, 205:475-488.

49. Breed MD, Moore J: Animal Behavior. Academic Press 2011.

50. Schumann RD, Buschinger A: Imprinting effects on host-selection behavior of slave-raiding Chalepoxenus muellerianus (Finzi) workers (Hymenoptera: Formicidae). Ethology 1995, 99:243-251.

51. Delattre O, Châline N, Chameron S, Lecoutey E, Jaisson P: Opportunist slave-making ants Myrmoxenus ravouxi discriminate different host species from a non-host species. Insect Soc 2012, doi:10.1007/s00040-012-0257-3. 
52. Ozaki M, Wada-Katsumata A, Fujikawa K, Iwasaki M, Yokohari F, Satoji Y, Nisimura T, Yamaoka R: Ant nestmate and non-nestmate discrimination by a chemosensory sensillum. Science (Washington, DC) 2005, 309:311-314.

53. Thompson JN: Coevolution: The geographic mosaic of coevolutionary arms races. Cur Biol 2005, 15:R992-994.

54. Nash DR, Als TD, Maile R, Jones GR, Boomsma JJ: A mosaic of chemical coevolution in a large blue butterfly. Science (Washington, DC) 2008, 319:88-90.

55. Bernard F: Note sur les fourmis de France. II Peuplement des montagnes méridionales. Ann Soc Ent France 1946, 115:1-36.

56. Bernard F: Faune de l'Europe et du Bassin Méditerranéen. Paris: Masson: Les fourmis (Hymenoptera Formicidae) d'Europe occidentale et septentrionale: 1967. 1968.

57. Buschinger A, Ehrhardt W, Fischer $K$, Ofer J: The slave-making ant genus Chalepoxenus (Hymenoptera, Formicidae). I. Review of literature, range, slave species. Zoologische Jahrbücher, Abteilung für Systematik, Ökologie und Geographie der Tiere 1988, 115:383-401.

58. Foitzik S, Heinze J: Nest site limitation and colony take over in the ant. Leptothorax nylanderi. Behav Ecol 1998, 9:367-375.

59. Provost $E$, Blight $O$, Tirard A, Renucci M: Hydrocarbons and insects' social physiology. In Insect Physiology: New Research. Edited by Maes RP.: Nova Science Publishers Inc; 2008:19-72.

60. Anderson MJ: A new method for non-parametric multivariate analysis of variance. Austral Ecol 2001, 26:32-46.

61. McArdle $\mathrm{BH}$, Anderson MJ: Fitting multivariate models to community data: a comment on distance-based redundancy analysis. Ecology 2001, 82:290-297.

62. Anderson MJ, Gorley RN, Clarke KR: PERMANOVA+ for PRIMER: guide to software and statistical methods. Plymouth, UK: PRIMER-E Ltd; 2008.

63. Clarke KR, Gorley RN: PRIMER v6: user manual/tutorial. Plymouth (UK): PRIMER-E Ltd; 2006.

64. Sprent P, Smeeton NC: Applied Nonparametric Statistical Methods. Chapman and Hall: $3 d$ ed; 2001.

65. Holm S: A simple sequentially rejective multiple test procedure. Scand J Statist 1979, 6:65-70.

66. Freeman $\mathrm{GH}$, Halton $\mathrm{JH}$ : Note on exact treatment of contingency, goodness of fit and other problems of significance. Biometrika 1951, 38:141-149.

67. Scherrer B: Biostatistiques, Volume 1, 2e édition. Montréal: Gaëtan Morin éditeur; 2007.

doi:10.1186/1742-9994-9-38

Cite this article as: Delattre et al:: Do host species evolve a specific response to slave-making ants?. Frontiers in Zoology 2012 9:38.

\section{Submit your next manuscript to BioMed Central and take full advantage of:}

- Convenient online submission

- Thorough peer review

- No space constraints or color figure charges

- Immediate publication on acceptance

- Inclusion in PubMed, CAS, Scopus and Google Scholar

- Research which is freely available for redistribution 